\title{
THE PREVALENCE OF VAGINAL CARRIER OF GROUP B STREPTOCOCCAL (GBS) AMONG PREGNANT WOMEN ATTENDING MATERNITY AT MUSTAFA- KHOMEINI HOSPITAL, ILAM
}

\author{
Jasem Mohamadi $^{1}$, rraj Pakzad $^{2}$, Zahra Khodai $^{3}$, Mohamad Reza Havasian ${ }^{4 *}$
}

${ }^{1}$ Department Pediatrics, Faculty of Medicine, Ilam University of Medical Sciences, Ilam, Iran
${ }^{2}$ Associate Professor, Department of Microbiology, Clinical Microbiology Research Center, Ilam University of Medical Sciences, Ilam, Iran
${ }^{3}$ School of Medicine, Ilam University of Medical sciences, Ilam, Iran
${ }^{4}$ Department of Periodontics, School of Dentistry, Ilam University of Medical sciences, Ilam, Iran

Received - May 01, 2017; Revision - August 20, 2017; Accepted - September 09, 2017

Available Online - September 10, 2017

http://dx.doi.org/10.18006/2017.5(4).561.567

KEYWORDS
Vaginal carrier
Group B Streptococcal
Aternity
Ilam
Iran

\begin{abstract}
The group B streptococcus or agalactiae streptococcus is gram positive bacteria. These organisms are normal flora of women genital and gastrointestinal tracts, which may cause early onset infection in new born infants and pregnant women. Severe infection of GBS may cause infection like meningitis, sepsis etc in new born infants. Determining the level of GBS carriage in the genital tract of pregnant mothers is useful for prophylaxis purposes and reducing the GBS infection. The present study was conducted on pregnant women, with pregnancy age of 35 weeks or more, who referred to Mustafa Khomeini, Ilam, from January 2014 to December 2015. Total 90 vaginal fluid samples were extracted from the vagina of pregnant women and Group B strep strains were identified by using cultivation methods. Antibiotic sensitivity of the samples were determined by disc-diffusion method. Data were analyzed using SPSS software, version 18. Chi-square and T-test were used as appropriated. Results of the present study revealed the incidence rate of Group B strep strains turned out to be $4.4 \%$ in pregnant women referring to Mustafa Khomeini, Ilam, during the study period. The Group B Strep colonization was also significantly associated $(\mathrm{P}<0.05)$ with the number of previous pregnancies. High prevalence of group $\mathrm{B}$ Streptococcus in pregnant women and their impact on the incidence of infection in both mothers and infants, screening is of crucial importance for pregnant women prior to giving birth. Considering high sensitivity of strains to Clindamycin, Vancomycin, and Cefazolin, it is recommended to consider these factors as the preferred treatment of infections caused by GBS.
\end{abstract}

* Corresponding author

E-mail: havasian1991@gmail.com, havasian-m@medilam.ac.ir (Mohamad Reza Havasian)

Peer review under responsibility of Journal of Experimental Biology and Agricultural Sciences.

Production and Hosting by Horizon Publisher India [HPI] (http://www.horizonpublisherindia.in/).

All rights reserved.
All the article published by Journal of Experimental Biology and Agricultural Sciences is licensed under a Creative Commons Attribution-NonCommercial 4.0 International License Based on a work at www.jebas.org.

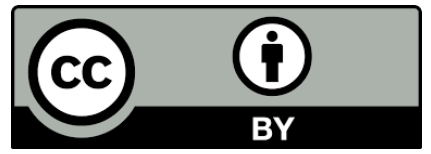




\section{Introduction}

Streptococci usually consider as an important pathologic species for humans. Some common streptococci species which infecting human being are Streptococcus pyogenes, S. agalactia, Enterococcus (None-Enterococcus), $S$. pneumonia and $S$. viridians. These species are gram positive and catalase negative cocci. Among these, Group B streptococcus ( $S$. agalactia) lived in the genetic tract of women's vaginal area and it is the main cause of invasive disease in pregnant women and newborns (Sarafrazi et al., 2001; Kwatra et al., 2014). Colonization is an asymptomatic phenomenon; however, bacteria can cause urinary tract infections, chorioamnionitis, endometritis, and Septicemia (Jahed et al., 2011). The colonization of GBS in the genetic tract of mother is most important risk factor for the neonatal and up to $70 \%$ of infants whose mothers were GBS colonized will be infected (Pirooz et al., 2000; Sarafrazi et al., 2001). In general, incidence rate of sepsis is 1 to 7 out of 10000 living infants (Sarafrazi et al., 2001). Further, various studies have shown that due time diagnosis of colonized babies and conducting Prophylaxis will reduce the incidence of neonatal sepsis up to 0.6 in each 10000 births (Jahed et al., 2011). Colonization and subsequent neonatal infection might occur in the womb, at birth, or in the first month of life. The incidence of this infection in first 7 days is referred to as 'premature' and it is called 'delayed' if it happens from the first week up to the first three months after the birth (Valkenburg -van et al., 2006).

Additionally, GBS colonization, especially in vagina and rectum, is a common phenomenon among pregnant women and $20-30 \%$ of the collected samples from pregnant women with pregnancy age of 35 weeks turn out to be GBS positive (Behrman et al., 2004). Colonization during pregnancy might occur in three forms i.e. chronic, transient, and intermittent (Behrman et al., 2004); consequently, samples obtained from vagina and rectum during 35 to 37 weeks of pregnancy can help in a reasonable prediction for the incidence of GBS colonization (Faxelius et al., 1988). According to laboratory and experimental findings, a streptococcal isolate can be examined through various biochemical tests, such as sodium Hippurate hydrolysis (where 99\% of isolates are positive), Bile escuiln agar hydrolysis (where 99 to $100 \%$ of isolates turn out to be negative), sensitivity to bacitracin test (where a 92\% resistance is produced), and CAMP factor production test (where 99 to $100 \%$ of isolates are positive) (Kasper et al., 2015).

This disease existence due to GBS colonization decrease in late 1990s due to predictive applications of antibiotics at the time of delivery; however, despite this promising trend, there were reported 2200 cases of infant infection in America in early days of their life (Lim et al., 1986). Group B Streptococcus infections occur more frequently in the adults in comparison to the infants while the incidence of diseases is more common in babies and pregnant women. Further, babies are more sensitive to GBS in comparison to men and non-pregnant women (Lim et al., 1986). Determining the amount of GBS vaginal infection in pregnant women can play a critical role in facilitated implementation of prophylaxis and reduction of these bacterial infections in infant's diagnosis of Rectovaginal GBS infection is quite important in the management of infant infection (Jahed et al., 2011; Konikkara et al., 2014; Brzychczy-Włoch et al., 2014).

There are different figures of GBS incidence in Iran; the incidence rate of GBS colonization is $26.7 \%$ in Hamedan while it was reported 9.2\% in Kerman. Further, in another study, $8 \%$ and $9.6 \%$ GBS colonization was reported by PCR method from these localities (Rabie et al., 2006; Aali et al., 2007; Bakhtiari et al., 2007; Fatemi et al., 2009). In this respect, present study has been carried out to estimate the prevalence of vaginal carrier of group B streptococcal among Pregnant Women attending to maternity Mostafa-Khomeini hospital, Ilam, Iran.

\section{Materials and Methods}

Vaginal samples were collected from 90 pregnant women with gestational age of 35 weeks and more and did not show any symptoms of prom or korio amniotes and had not taken any antibiotic. Further required information was extracted through the well prepared questionnaire.

\subsection{Isolation and Maintenance of Isolates}

The isolated vaginal samples were collected in PBS and Stuart's Medium (CONDA, Spain) and transferred to laboratory for culturing; then, they were set in Toddhewibroht medium (CONDA, Spain), which contained $10 \mathrm{mg} / \mathrm{l}$ Gentamicin and 15 $\mathrm{mg} / \mathrm{l}$ Nalidixic acid; then the samples were cultured in Agar blood medium, containing 5\% sheep blood, after 24 hours incubation at $37^{\circ} \mathrm{C}$ temperature; finally, the samples were re-incubated at $37^{\circ} \mathrm{C}$ in an incubator containing $5 \% \mathrm{CO}_{2}$. Culturing was followed by the identification based on the predefined CBS characteristics such as Gram-positive, catalase negative, beta-hemolysis cocci, this made possible by conducted tests including bacitracin, Bile Esculin Agar non-hydrolysis, and cAMP, were implemented in order to recognize GBS; then, GBS isolates were stored in TBS medium (CONDA, Spain) containing $10 \%$ glycerol at $20^{\circ} \mathrm{c}$ temperature (Mohamadi et al., 2014).

\subsection{Determining Microbe Sensitivity}

Initially, 3-5 specimens colony were collected and transferred into TBS medium; these collected samples were incubated for 2-6 hours at $37^{\circ} \mathrm{C}$ temperature in suspension conditions. Then, cell suspension was prepared as per the McFarland concentration standard. This was followed by the extraction of pure culture of 
microbes by Swab and cultured in Mueller-Hinton agar medium containing 5\% sheep blood. After 3-5 minutes, antibiotic marked discs were kept in bacterial culture containing Petri plate. The intended plates were incubated for $16-18$ hours at $37^{\circ} \mathrm{C}$ temperature; then, the diameter of protective halo was measured and labeled as sensitive, semi-sensitive, non-sensitive according to results. To determine antibiotic sensitivity, Penicillin, Ampicillin, Erythromycin (25\% and 75\%), Ampicillin (25\%), Cefazolin, Clindamycin, and Vancomycin were used as a test antibiotic (Havasian et al., 2013; Panahi et al., 2013).

\subsection{Statistical analysis}

Data were analyzed using SPSS software, version 18. Chi-square and T-test were used as appropriated (Havasian et al., 2012;
Havasian et al., 2014).

\section{Results}

In present study 90 pregnant women, with mean age of 27 years were examined. Most of the studied females were housewives and did not have any history of abortion or any other chronic disease. Among the studied female, $66.7 \%$ belongs to age group 20-30 years, the youngest being was 16 while the oldest one was 42 years old (Table 1). The mean age of the patients with positive culture was $29.75 \pm 4.34$ years women who had associate degree has the highest frequency of positive culture cases $(\mathrm{P}>0.05)$. In case of residing, women who stayed in cities have highest frequency of positive culture cases as compared to the women's residing in villages, these two categories are significantly different

Table 1 Frequency Distribution of demographic characteristics of studied patients

\begin{tabular}{|c|c|c|c|c|}
\hline \multicolumn{2}{|c|}{ Variable } & Frequency & Percent & P-value \\
\hline \multirow{2}{*}{ Job } & Housewife & 77 & 85.6 & \multirow{2}{*}{1.1} \\
\hline & Practitioner & 13 & 14.4 & \\
\hline \multirow{3}{*}{ Education } & Under diploma & 54 & 60 & \multirow{3}{*}{0.94} \\
\hline & Diploma & 17 & 18.9 & \\
\hline & Above diploma & 19 & 21.1 & \\
\hline \multirow{2}{*}{ Location } & Urban & 77 & 85.6 & \multirow{2}{*}{0.84} \\
\hline & Rural & 13 & 14.4 & \\
\hline \multirow{4}{*}{ Number of Deliveries } & 1 & 45 & 50 & \multirow{4}{*}{0.04} \\
\hline & 1 & 26 & 28.9 & \\
\hline & 3 & 14 & 15.6 & \\
\hline & 4 & 5 & 5.6 & \\
\hline \multirow{3}{*}{ Number of Abortions } & $\mathbf{0}$ & 70 & 77.8 & \multirow{3}{*}{0.59} \\
\hline & 1 & 17 & 18.9 & \\
\hline & 2 & 3 & 3.3 & \\
\hline \multirow{6}{*}{ Underlying Disease } & None & 67 & 74.4 & \multirow{6}{*}{0.22} \\
\hline & Gestational diabetes & 11 & 12.2 & \\
\hline & Hypothyroidism & 2 & 2.2 & \\
\hline & Iron deficiency anemia & 6 & 6.7 & \\
\hline & Hyperthyroid & 1 & 1.1 & \\
\hline & Pre-eclampsia & 3 & 3.3 & \\
\hline \multirow{2}{*}{ Preterm Delivery } & Yes & 7 & 7.8 & \multirow{2}{*}{1} \\
\hline & No & 83 & 92.2 & \\
\hline
\end{tabular}


to each other $(\mathrm{P}>0.05)$. Further, results of study suggested that frequency of GBS positive culture cases were increased with the increasing the number of delivery (Table 2) and highest frequency was related to women who were experiencing their $3^{\text {rd }}$ delivery $(\mathrm{P}>0.05)$. It was also reported that $5.9 \%$ of the patients with positive culture had at least one experience of abortion. Further it was reported that $50 \%$ of the positive cultures patients had gestational diabetes $(\mathrm{P}<0.05)$. There was no significant relationship reported between positive culture and history of delivery preterm $(\mathrm{P}>0.05)$. Patients with positive culture turned out to be semi sensitive to Penicillin, Ampicillin, and Erythromycin (75\%) and resistant to Ampicillin (25\%), Penicillin, and Erythromycin (25\%); they, also, turned out to be quite sensitive to Cefazolin, Clindamycin, and Vancomycin (Table 3).
However, GBS infection has grown more seriously in adults suffering from chronic diseases and nowadays the adults make up a larger proportion of invasive GBS infections in comparison to infants (Kasper et al., 2015). The incidence rate of GBS in the genital tract of pregnant women varies from 5 to $40 \%$ in different geographical locations (Madzivhandila et al., 2011). GBS colonization in genital system causes unpleasant repercussions and complication such as preterm labor, preterm rupture of membrane, clinical chorioamnionitis, subclinical chorioamnionitis, endometriosis, and many other post labor and neonatal infection, at the time of delivery for both mother and infant (Behrman et al., 2004; Faxelius et al., 1988; James et al., 2005). The infection resulting from GBS in preterm infants, who have been exposed to uterine, vaginal, or rectal infection, is,

Table 2 Determining the incidence rate of Vaginal Group B Streptococcus in pregnant women in terms of the number of deliveries

\begin{tabular}{|c|c|c|c|c|}
\hline \multirow{3}{*}{ Number of Deliveries } & \multicolumn{4}{|c|}{ Culture } \\
\hline & \multicolumn{2}{|c|}{ Positive } & \multicolumn{2}{|c|}{ Negative } \\
\hline & Frequency & Percent & Frequency & Percent \\
\hline 1 & 0 & 0 & 45 & 100 \\
\hline 2 & 1 & 3.8 & 25 & 96.2 \\
\hline 3 & 2 & 14.3 & 12 & 85.7 \\
\hline 4 & 1 & 20 & 4 & 80 \\
\hline Total & 4 & 4.4 & 86 & 95.6 \\
\hline P-value & \multicolumn{4}{|c|}{0.043} \\
\hline
\end{tabular}

Table 3 Frequency distribution of the analysis of anti-biogram results in positive culture samples

\begin{tabular}{|c|c|c|c|c|c|c|c|c|}
\hline \multirow[t]{2}{*}{ Anti-biogram } & \multicolumn{6}{|c|}{ Results } & \multicolumn{2}{|c|}{ Total } \\
\hline & Frequency & Percent & Frequency & Percent & Frequency & Percent & Frequency & Percent \\
\hline Penicillin & 3 & 75 & 1 & 25 & 0 & 0 & 4 & 100 \\
\hline Ampicillin & 3 & 75 & 0 & 0 & 1 & 25 & 4 & 100 \\
\hline cefazolin & 4 & 100 & 0 & 0 & 0 & 0 & 4 & 100 \\
\hline Erythromycin & 3 & 75 & 1 & 25 & 0 & 0 & 4 & 100 \\
\hline Clindamycin & 4 & 100 & 0 & 0 & 0 & 0 & 4 & 100 \\
\hline Vancomycin & 4 & 100 & 0 & 0 & 0 & 0 & 4 & 100 \\
\hline
\end{tabular}

\section{Discussion}

GBS is a common cause of peripartum fever in pregnant women and occasionally it caused serious infections in nonpregnant adults. GBS oriented prenatal screening decreased the rate of neonatal infections from 6-7 cases to 0.6 in each 1000 live births. sometimes, revealed from 24 hours to one whole week after the birth. Initially, the lungs are infected and, then, septicemia and meningitis might result in death in $70-75 \%$ of cases (Edwards \& Baker, 2000). Incidence rate of GBS turned out to be $4.4 \%$ in pregnant women referring to hospitals in Ilam; this rate was $20.4 \%$ in Brazil, $17.9 \%$ in Italy, $9.6 \%$ in Tabriz, $14 \%$ in Khurram 
Abad and 13.2\% in Ahvaz (Shahbazian et al., 2007; Nazer et al., 2011; AbdollahiFard et al., 2008). In a study conducted on 524 pregnant women in India, $4.77 \%$ of patients turned out to be GBS colonization positive (Dechen et al., 2010); this difference in incidence rate might be due to difference in site of biopsy, diagnostic methods, study populations, and ethnic and geographic variations. According to the results of the present study, there was no significant relationship between vaginal GBS colonization and education, residence, number of abortions, subsidiary diseases, preterm labor, and occupation; however, this turned out to be a positive relationship between GBS colonization and the number of deliveries. Similarly, Nazr et al. (2011) and Shahbazian et al (2007) did not reported any significant relationship between the age of the mother or gestational age and GBS colonization in Khurram Abad and Ahvaz respectively (Shahbazian et al., 2007; Nazer et al., 2011). Further in the present study a significant relationship was reported between the GBS colonization and the number of deliveries which was inconsistent with the results of the study conducted by Nahaei et al (2007), on 965 pregnant women in Tabriz, where vaginal colonization incidence rate was reported to be $2.5 \%$. This inconsistency of results might be due to difference in studies population and the limited number of mothers with a history of former pregnancies. The results of the study conducted in Khurram Abad showed a significant relationship between colonization and the number of deliveries (Nazer et al., 2011), which is consistent with the findings of the present study. Further no significant relationship was reported between the results of culture analysis and underlying diseases. Based on the results of a research conducted in Mashhad, comparison of GBS incidence rate in diabetes and non-diabetes women revealed that rate of vaginal GBS colonization turned to be $11.8 \%$, which turned out to be higher in diabetes women as compared to the non-diabetes ones (Akhlaghi et al., 2009). Chu et al (2007) conducted a study, in Hong Kong and reported that isolated Group B Streptococcus turned out to be $100 \%$ sensitive to vancomycin and 4-strained GBS showed less sensitivity to penicillin (Akhlaghi et al., 2009). Mandell et al. (2005) conducted a study, the antibiotic sensitivity of the strains, all strains $100 \%$ sensitive to Penicillin and Ampicillin, was measured using discdiffusion method; $29.6 \%$ of the strains were sensitive to clindamycin and $42.1 \%$ were sensitive to erythromycin (Mandell et al., 2005). Difference in the results of the present study and other studies conducted on the same issue might be due to difference in approaches to the studied antibiotics. There was significant relationship between colonization and preterm labor; this inconsistency may be due to the limited number of mothers who participated in the present study and had a history of preterm labor. However, there was no significant relationship between rate of colonization (21\%) and age of the mother and preterm labor in Valkenburg-van et al. (2006) in Netherlands; these results are consistent with the findings of the present study (Valkenburg-van et al., 2009).

\section{Conclusion}

Result of this study revealed a significant relationship between the incidence rate of colonization and the number of former deliveries. Further, it is highly recommended for the mothers with a history of disease that they should be strictly monitored before labor. Also, considering the high incidence rate of GBS in pregnant women who live in Ilam and its danger as a risk factor which might results in mother and neonatal infections, it is highly recommended for pregnant women to screen through vaginal culture for GBS in order to provide early treatment and prevent transmission to newborns. Considering high sensitivity of isolated strains to clindamycin, vancomycin, and cefazolin, it is recommended to consider possible impact of these drugs to cure infections resulting from GBS.

\section{References}

Aali BS, Abdollahi H, Narkhaee N, Davazdahemami Z, Mehdizadeh A (2007) The association of preterm labor with vaginal colonization of group B streptococci. Iranian Journal of Reproductive Medicine 5: 191-94.

AbdollahiFard S, Ghotasloo R, Zafardoost S (2008) Study on colonization of group B streptococcus (GBS) and relationship with perinatal complication in pregnant women referred to Alzahra hospital. Research Journal of Biological Sciences 3: 726728.

Akhlaghi F, Hamedi A, NaderiNasab M (2009) Comparison of Group B Streptococcal Colonization in the Pregnant Diabetic and Non-Diabetic Women. Acta Medica Iranica 47: 103-08.

Bakhtiari R, SoltanDallal MM, ZaemiYazdi MJ, Fallah J, Amir Mozaffari N, Pourmand MR (2007) Evaluation of PCR method for diagnosis of Group B Streptococcus carriage in pregnant women. Iranian Journal of Medical Microbiology 1: 1-8.

Behrman RE, Kliegman RM (2004) Nelson Textbook of Pediatrics. $17^{\text {th }}$ ed. Elsevier Saunders, Philadelphia Pp. 879-83.

Mandell GL, Bennett JE, Dolin R (2005) editors. Principles and Practice of Infection Diseases. 6th ed. Churchill Livingstone, Elsevier, Pp. 2425-2427.

Brzychczy-Włoch M, Pabian W, Majewska E, Zuk MG, Kielbik J, Gosiewski T, Bulanda MG (2014) Dynamics of colonization with group $\mathrm{B}$ streptococci in relation to normal flora in women during subsequent trimestersof pregnancy. 
New Microbiologica 37: 307-319.

Dechen TC, Sumit K, Ranabir P (2010) Correlates of vaginal colonization with group B streptococci among pregnant women. Journal of Global Infectious Diseases 2: 236-41.

Chu YW, Tse C, Tsang GK, So DK, Fung JT, Lo JY (2007) Invasive group B Streptococcus isolates showing reduced susceptibility to penicillin in Hong Kong. Journal of Antimicrobial Chemotherapy 60: 1407-09.

Edwards M, Baker CJ (2000) Streptococcus ahalsctia. In: Mandell GI (Ed) Bennetts Principles practices of infectious Disease, Sthed, Vol 4. Churchill -Living stone, New York, Pp. 2156-2164.

Fatemi F, Chamani L, Pakzad P, Zeraati H, Rabbani H, Asgari S (2009) Colonization rate of group B Streptococcus (GBS) in pregnant women using GBS agar medium. Acta medica Iranica 47: $25-30$

Faxelius G, Bremme K, Kvist-Christensen K, Christensen P, Ringertz S (1988) Neonatal septicemia due to group B streptococci--perinatal risk factors and outcome of subsequent pregnancies. Journal of Perinatal Medicine 16: 423-30.

Havasian MR, Panahi J, Khosravi A (2012) Correlation between the lipid and cytokine profiles in patients with coronary heart disease (CHD). Life Science Journal 9: 5772-77.

Havasian MR, Panahi J, Mahdieh N (2014) Cystic fibrosis and distribution and mutation analysis of CFTR gene in Iranian patients. Koomesh 15: 431-40.

Havasian MR, Panahi J, Pakzad I, Davoudian A, Jalilian A, ZamanianAzodi M (2013) Study of Inhibitory effect of alcoholic and aqueous extract of Scrophulariastriata (tashnedari) on candida albicans in vitro. Research in Medicine 36: 19-23.

Jahed T, KhoshnoodShariati M, Zafarghandi A, Darabi P, Karimi A (2011) Frequency of Group B Streptococcus colonization and antibiogram in women at 35-37 weeks of gestation visited in prenatal clinic of Mahdieh Hospital in 2008. Pejouhandeh 16: 3943.

James DK, Steer PH, Weiner CP, Gonik B (2005) High Risk Pregnancy Management option. 3rd ed. Elsevier Saunders, Philadelphia Pp. 674-90.

Kasper D, Fauci A, Hauser S, Longo D, Jameson J, Loscalzo J (2015) Harrison's principles of internal medicine, $19^{\text {ed }}$ The McGraw-Hill publication.
Konikkara KP, Baliga S, Shenoy S, Bharati B (2014) Evaluation of culture, antigen detection and polymerase chain reaction for detection of vaginal colonization of group B Streptococcus (GBS) in pregnant women. Journal of Clinical and Diagnostic Research 8: 47-49.

Kwatra G, Adrian PV, Shiri T, Buchmann EJ, Cutland CL, Madhi SA (2014) Serotype-Specific Acquisition and Loss of Group B Streptococcus Recto-Vaginal Colonization in Late Pregnancy. Plos one 9: $98778 . \quad$ DOI: https://doi.org/10.1371/journal.pone.0098778.

Lim DV, Morales WJ, Walsh AF, Kazanis D (1986) Reduction of morbidity and mortality rates for neonatal Group B Streptococcal disease through early diagnosis and chemoprophylaxis. Journal of Clinical Microbiology 23: 489-92.

Madzivhandila M, Adrian PV, Cutland CL, Kuwanda L, Schrag SJ, Madhi SA (2011) Serotype distribution and invasive potential of group B streptococcus isolates causing disease in infants and colonizing maternal-newborn dyads. PLoS One 6: 17861-68. DOI: https://doi.org/10.1371/journal.pone.0017861.

Mohamadi J, Motaghi M, Panahi J, Havasian MR, Delpisheh A, Azizian M, Pakzad I (2014) Anti-fungal resistance in candida isolated from oral and diaper rash candidiasis in neonates. Bioinformation 10: 667-70.

Nahaei MR, Ghandchilar N, Bilan N, Ghahramani P (2007) Maternal carriage and neonatal colonization of Streptococcus agalactiae in Tabriz, Northwest Iran. Iranian Journal of Medical Sciences 32: 177-81.

Nazer MR, Rafiei Alavi E, Nazer E, Khamechi M (2011) Prevalence of Group B Streptococcus Vaginal Colonization in the Third Trimester of Pregnancy. Journal of Shahid Sadoughi University of Medical Sciences 19: 13-23.

Panahi J , Havasiyan MR, Gheitasi S, Pakzad I, Jaliliyan A, Hoshmandfar R, Havasiyan M (2013) The in Vitro Inhibitory Effects of the Aqueous Extracts of Summer Onion on Candida albicans. Journal of Ilam University of Medical Sciences 21: 5459 .

Pirooz T, Farsi S, ForooheshTehrani H (2000) Group B Streptococci colonization in Pregnant Women In Labor. Razi Journal of Medical Sciences 6: 275-80.

Rabie S, Arab M, YousefiMashouf R (2006) Epidemiologic Pattern of Vaginal Colonization by group $\mathrm{B}$ streptococcus in pregnant women in Hamadan, Central west of Iran. Iranian Journal of medical Sciences 31: 106-08. 
Sarafrazi N, Mesdaghinia E, Moniri R, Mousavi SGA (2001) Evaluation of vaginal Streptococcus hemolytic type B in pregnant women and its relationship with early neonatal infection. Feyz Journal of Kashan University of Medical Sciences 5: 22-7.

Shahbazian N, Rajabzadeh AR, Alavi M (2007) Prevalence of Group -BStreptococcal Colonization in Vagina and Rectum of 35-37 Weeks Pregnant Women and its Sensitivity to Antibiotics. Journal of Ahwaz University Medical Sciences 6: 294-98.
Valkenburg-van den Berg AW, Sprij AJ, Dekker FW, Dprr PJ, Kanhai HH (2009) Association between colonization with group B streptococcus and preterm delivery: a systematic review. Acta Obstetricia et Gynecologica Scandinavica 88: 958-966.

Valkenburg-van den Berg AW, Sprij AJ, Oostvogel PM, Mutsaers JA, Renes WB, Rosendaal FR, Joep Dörr P (2006) Prevalence of colonisation with group B Streptococci in pregnant women of a multi-ethnic population in The Netherlands. European Journal of Obstetrics \& Gynecology and Reproductive Biology 124: 178-83. 